\title{
Characterization of Nondiffusive Transport in Plasma Turbulence via a Novel Lagrangian Method
}

\author{
J. A. Mier, ${ }^{1, *}$ R. Sánchez, ${ }^{2}$ L. García, ${ }^{1}$ B. A. Carreras, ${ }^{3}$ and D. E. Newman ${ }^{4}$ \\ ${ }^{1}$ Departamento de Física, Universidad Carlos III de Madrid, 28911 Leganés, Madrid, Spain \\ ${ }^{2}$ Fusion Energy Division, Oak Ridge National Laboratory, Oak Ridge, Tennessee 37831-8071, USA \\ ${ }^{3}$ BACV Solutions, Inc., Oak Ridge, Tennessee 37830-8222, USA \\ ${ }^{4}$ Department of Physics, University of Alaska, Fairbanks, Alaska 99775-5920, USA
}

(Received 18 June 2008; published 13 October 2008)

\begin{abstract}
A novel method to probe and characterize the nature of the transport of passive scalars carried out by a turbulent flow is introduced. It requires the determination of two exponents which encapsulate the statistical and correlation properties of the component of interest of the Lagrangian velocities of the flow. Numerical simulations of a magnetically confined, near-critical turbulent plasma, known to exhibit superdiffusive radial transport, are used to illustrate the method. It is shown that the method can easily detect the change in the dynamics of the radial transport that takes place after adding to the simulations a (subdominant) diffusive channel of tunable strength.
\end{abstract}

DOI: 10.1103/PhysRevLett.101.165001

In the last few decades, a large amount of experimental evidence has shown that in many systems of interest the transport of various quantities (such as mass, energy, reactants, pollutants, etc.) often exhibits a nondiffusive character [1]. The resulting transport-also referred to using terms such as anomalous, scale-free, or fractional-appears to be well modeled in terms of differential equations which, in contrast to the usual diffusion equation, contain fractional derivatives [2]. The simplest one is

$$
\frac{\partial n}{\partial t}={ }_{0} D_{t}^{1-\beta}\left[C \frac{\partial^{\alpha} n}{\partial|x|^{\alpha}}\right],
$$

where ${ }_{a} D_{t}^{\gamma}$ represents the fractional Riemann-Liouville derivative of order $\gamma$ and start point $a$, and $\partial^{\delta} n / \partial|x|^{\delta}$ is the Riesz fractional derivative of order $\delta . C$ is a so-called fractional diffusivity. Typically, $\beta \in(0,1)$ and $\alpha \in(0,2)$.

The definition of a fractional derivative will not be needed in what follows (it can be found elsewhere [2]). It suffices to say that it is an integro-differential operator which contains integrals over the past history or the extension of the system. To understand what their presence in Eq. (1) implies, it is useful to remember that diffusive transport is exhibited whenever typical length $(l)$ and time $(\tau)$ transport scales exist in the system (much smaller than the system size and lifespan). Beyond these typical scales, the underlying microscopic dynamics behave essentially as a Gaussian and Markovian process. Consequently, transport is well described by diffusive equations, the simplest of them being $\partial_{t} n=D \partial_{x}^{2} n$, with a diffusivity $D \sim l^{2} / \tau$. In contrast, in situations where nondiffusive transport is observed either a typical length scale or a typical time scale or both are usually lacking. Deviations from Gaussianity and Markovianity should then be expected. In fact, the necessity of fractional operators is due to these deviations and reflects the fact that every length and time scale participates now in setting the
PACS numbers: 52.35.Ra, 05.40.Fb, 47.27. $-\mathrm{i}, 52.65 . \mathrm{Kj}$

character of macroscopic transport. Since Eq. (1) reduces to the diffusive equation when $\beta \rightarrow 1$ and $\alpha \rightarrow 2$, any deviation from Gaussianity and Markovianity can be detected and quantified if the values of these exponents can be determined in practical situations. Once known, appropriate fractional equations become available, which should be very useful in many areas of science and engineering [1]. For this reason, finding simple, efficient, and reliable methods to estimate them is of great importance.

Of particular interest to us are cases in which nondiffusive transport appears in the context of systems governed by turbulence. This is not a rare situation. For instance, it is often observed in magnetically confined toroidal plasmas, such as those inside a tokamak [3]. It has been proposed that the cause might be related to the fact that the density and temperature radial profiles are continually pushed beyond their local instability thresholds by the external heating and fueling, only to be brought again below them by the radial transport driven by the ensuing turbulence [4]. The dynamics of this near-critical regime are such that proper typical length and time scales are no longer defined, which would yield nondiffusive radial transport. Some experimental support for these ideas already exists, since both radial avalanches and long-term temporal correlations have been reported [5], but remains controversial. Most of the evidence available comes instead from numerical simulations of various types of plasma turbulence [6-8]. In them, the determination of the aforementioned exponents is, however, a long, subtle, and complicated procedure.

In this Letter we introduce a simple and robust method to obtain these exponents in any simulation of either fluid or plasma turbulent flows. As will be shown, the method is not hampered by the usual limitations encountered in these type of simulations (namely, small number of tracers and/ or short temporal records), which should also make it particularly suitable for real experiments. The method 
follows directly from recent theoretical work [9] which shows that, for a passive scalar (transport of active scalars may, however, be different due, for instance, to inertial effects [10]) and in the absence of a mean flow, $\alpha$ and $\beta$ can be determined from the statistical and correlation properties of the component of interest of the flow Lagrangian velocity. For instance, this would be the radial component in the case of tokamaks. In simulations, the Lagrangian velocity time series can be obtained by introducing tracer particles, which are then advected by the flow along the trajectory $\dot{\mathbf{R}}\left(t \mid \mathbf{r}_{0}, t_{0}\right)=\mathbf{V}(\mathbf{R}, t)$, once the initial condition $\mathbf{R}\left(t_{0} \mid \mathbf{r}_{0}, t_{0}\right)=\mathbf{r}_{0}$ is prescribed. $\mathbf{V}(\mathbf{r}, t)$ is the flow field. We proceed now to detail how each exponent is computed from a preexistent set of Lagrangian velocity time series of the desired component (presumably obtained with many different $\mathbf{r}_{0}$ 's and $t_{0}$ 's). An example of application follows.

As shown in Ref. [9], the exponent $\alpha$ is determined by the statistics of the Lagrangian velocity component averaged over many realizations, after removing any existing mean flow. This is so because they are typically distributed according to some stable law that satisfies the central limit theorem [11]: either a Gaussian law or a Lévy symmetric distribution. In contrast to the more familiar Gaussian, Lévy laws decay as power laws with exponent $1+\alpha$ with $\alpha \in(0,2)$, which sets $\alpha$. This algebraic decay causes Lévy laws to have an infinite variance (in fact, all moments of order $s \geq \alpha$ are infinite). This property can be related to the absence of typical transport scales in the flow, and explains the range of $\alpha$ 's in Eq. (1). In contrast, all moments of the Gaussian are finite.

The second exponent, $\beta$, is not computed directly but using $\beta=\alpha H_{\alpha}$, where $H_{\alpha}$ is the $\alpha$-Hurst exponent of the Lagrangian velocity component time series, averaged over many realizations [9]. The standard Hurst exponent $\left(\mathrm{H}_{2}\right.$, in our notation) has been used for years to quantify correlations in ordered, stationary series with Gaussiandistributed entries [12]. To explain why, it suffices to say that, in one of its interpretations, $\mathrm{H}_{2}$ is also the selfsimilarity exponent of the path constructed by using the series entries as successive increments [13]. Since the selfsimilarity exponent of the standard random walk is $1 / 2$, the estimation of $\mathrm{H}_{2}$ can reveal whether the series entries are correlated or not: if $H_{2}=1 / 2$ they are uncorrelated, if $H<1 / 2$, they are anticorrelated and, if $H_{2}>1 / 2$, the correlation is positive. Analogously, $H_{\alpha}$ is the selfsimilarity exponent of the path constructed using instead entries distributed according to a symmetric Lévy law. The entries are uncorrelated if $H_{\alpha}=1 / \alpha$, anticorrelated for $H_{\alpha}<1 / \alpha$ and positively correlated otherwise.

There are many ways to compute the Hurst exponent. One is to construct the rescaled range [12] of the (numerically discrete) time series, $v(t) \rightarrow\left\{V_{i}, i=1, \ldots, N\right\}$ :

$$
[R / S](\tau) \equiv \frac{\max _{1 \leq k \leq \tau} W(k, \tau)-\min _{1 \leq k \leq \tau} W(k, \tau)}{\sqrt{\left\langle V^{2}\right\rangle_{\tau}-\langle V\rangle_{\tau}^{2}}} .
$$

Here, $W(k, \tau) \equiv \sum_{i=1}^{k} V_{i}-k\langle V\rangle_{\tau}$ and $\langle\cdot\rangle_{\tau}$ represents the average up to time $\tau$. When the signal is self-similar, $[R / S] \sim \tau^{H_{2}}$ over some range of scales and $\mathrm{H}_{2}$ is the Hurst exponent. The prescription must, however, be changed for Lévy-distributed entries. Indeed, since their variance is infinite, the denominator of the rescaled range would scale with $\tau$ distorting the exponent, which would no longer be the self-similarity exponent. The fix is easy: substitute the square root of the variance by the $1 / s$ th power of any moment of order $s>0$, with $s<\alpha$.

To illustrate the method, we apply it now to a set of fluid simulations of a magnetically confined cylindrical plasma in which superdiffusive radial transport has been observed previously [14]. The turbulence is electrostatic and driven by the dissipative trapped electron mode (DTEM). Furthermore, the description of the relevant dynamics is reduced, by considering its long-wavelength limit, to a pair of coupled partial differential equations, respectively, describing the time evolution of the mean and fluctuating ion densities [15]. Because of limitations in space, we do not include the equations here. They can be found in Ref. [14], together with a discussion of the physics they contain. (Note that the simulations discussed here are based on the same equations but use different parameters and profiles.) Since fluctuating and mean ion densities are coupled self-consistently in these runs, the system can be made to exhibit superdiffusive radial transport by driving it so that the mean density profile is continuously pushed beyond its instability threshold, as pointed out previously. What makes these simulations particularly suitable is that the nature of radial transport can be made to become closer to diffusive by including an additional diffusive channel and increasing its intensity from zero $[14,16]$. This change occurs in spite of the fact that most of the transport is still carried out by turbulence. Ultimately, the change in dynamics should be due to a shift towards Gaussianity and Markovianity at a more microscopic level. For this reason, these simulations provide a good testbed to illustrate how the Lagrangian method quantifies different transport dynamics.

The geometry of the simulations is shown in Fig. 1. It is a periodic cylinder with minor radius $a=0.5 \mathrm{~m}$ and length $2 \pi R_{0}$, with $R_{0}=2 \mathrm{~m}$. Each location is labeled ( $r$, $\theta, \phi)$ being $r$ the radius normalized to $a$, and $\theta$ and $\phi$ the poloidal and toroidal angles. The confining magnetic field has an axis value $B_{0}=1 \mathrm{~T}$ and safety factor profile: $q(r)=1.3+0.5 r^{2}$. The plasma is deuterium, with cold ions and hot electrons and using parameter values typical of tokamak discharges. The density and electron temperature profiles are $n(r)=n_{0}\left(1-r^{2}\right)$ and $T_{e}=T_{0}\left(1-r^{2}\right)^{2}$, so that the DTEM instability parameter $\eta_{e}(r) \equiv$ $d\left(\ln T_{e}\right) / d(\ln n)=2$ all across the radius. Axis values are $n_{0}=10^{19} \mathrm{~m}^{-3}$ and $T_{0}=2.5 \mathrm{keV}$. The region that contains the $(\sim 150)$ modes susceptible of becoming unstable and where turbulence ensues is the interval $\left[r_{\mathrm{in}}, r_{\mathrm{out}}\right]=$ $[0.48,0.75]$. In physical units, its size is $L=135 \mathrm{~mm}$. To 


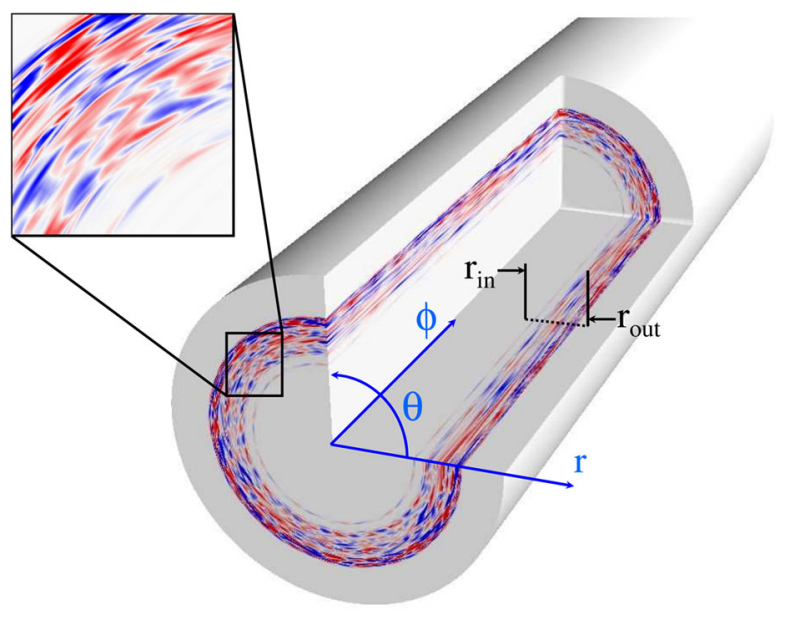

FIG. 1 (color online). Geometry of simulations. Inset: snapshot of density fluctuation contours [red (gray) $\rightarrow$ positive; blue (dark gray) $\rightarrow$ negative].

ensure convergence, the radial grid size is $\Delta r=0.25 \mathrm{~mm}$ and the temporal step is $\Delta t=1 \mu \mathrm{s}$. For reference purposes, note that the ion Larmor radius is $\rho_{L} \sim 4 \mathrm{~mm}$; the turbulence radial decorrelation length, $l \sim 15 \mathrm{~mm}$; the inverse growth rate of the most unstable mode is $\gamma_{L}^{-1} \sim$ $65 \mu \mathrm{s}$; the Eulerian and Lagrangian turbulent decorrelation times are $\tau_{E} \sim 40 \mu \mathrm{s}$ and $\tau_{L} \sim 5 \mu \mathrm{s}$; finally, each simulation is $\sim 50 \mathrm{~ms}$ long.

The time series of Lagrangian radial velocities required are obtained using tracers [7,8], which are advected by the $\mathbf{E} \times \mathbf{B}$ drift obtained from the time evolving turbulence using: $\dot{\mathbf{R}}=[\mathbf{E}(\mathbf{R}, \mathbf{t}) \times \mathbf{B}(\mathbf{R})] /|\mathbf{B}(\mathbf{R})|^{2}$. The electric field $\mathbf{E}$ is computed from the fluctuating electrostatic potential $\phi$ which, in this DTEM model, is determined from the density fluctuations [15]. The tracers are randomly initialized within $\left[r_{\text {in }}, r_{\text {out }}\right]$ and when their trajectories leave the interval, they are randomly relocated within, but without losing their individual identity. Four simulations have been examined, corresponding to four different values of the diffusivity $\left(D_{0}\right)$ that sets the intensity of the additional diffusive channel, all of them sufficiently small so that most of the radial transport is still carried by the turbulence. In Fig. 2, the probability density functions of the radial Lagrangian velocities are shown, together with the best (in a chi-square sense) symmetric Lévy fit. In Fig. 3, the "instantaneous" $\alpha$-Hurst exponent is estimated using $H_{\alpha} \sim(\tau /[R / S]) d[R / S] / d \tau$. To interpret it, it must be noted that the self-similar range of time scales over which $H_{\alpha}$ is meaningful extends from the local turbulent scales (here, $\tau_{L} \sim 5 \mu \mathrm{s}$ ) up to the mean tracer confinement time. No correlation can be maintained beyond that time since tracers exiting the region are randomly relocated within. This maximum time scale shows as a sudden drop towards the uncorrelated value $(1 / 2)$, and is consistent with the confinement time computed directly from the tracers. (The drop disappears for the largest $D_{0}$ because, as discussed next, the dynamics are already almost diffusive and thus

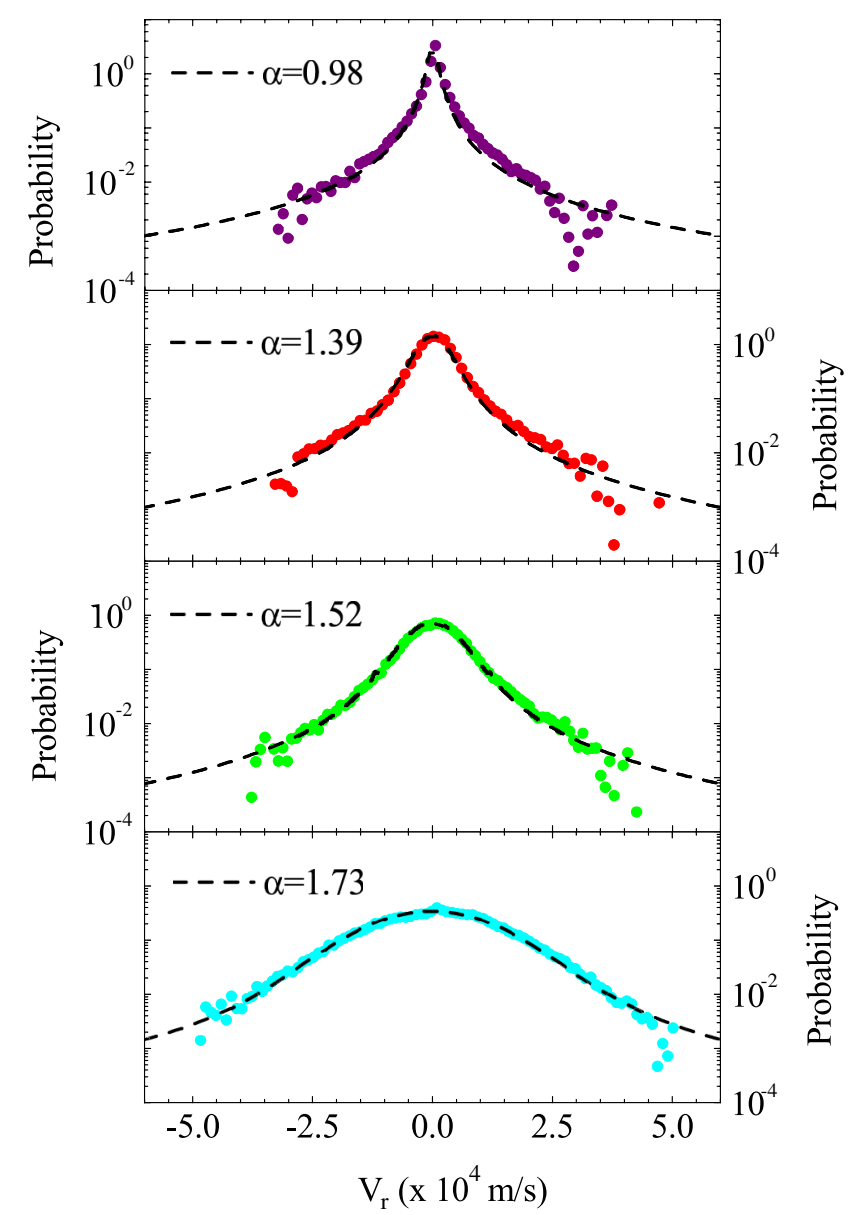

FIG. 2 (color online). Probability density functions of Lagrangian velocities for various diffusivities (top: $D_{0}=0$; second: $D_{0}=0.07 \mathrm{~m}^{2} / \mathrm{s}$; third: $D_{0}=0.18 \mathrm{~m}^{2} / \mathrm{s}$; bottom: $D_{0}=$ $1.19 \mathrm{~m}^{2} / \mathrm{s}$ ). Best Lévy fit shown in dashed black lines.

uncorrelated.) These times are collected in Table I, together with the values of $\alpha, H_{\alpha}$, and $\beta$ obtained for all cases. In the absence of the diffusive channel $\left(D_{0}=0\right)$, $H_{\alpha} \simeq 0.74, \alpha \simeq 0.98$, and $\beta=0.73$. That is, radial transport is superdiffusive but, as expected, also strongly nonGaussian and non-Markovian. More interestingly, the method clearly detects and quantifies the gradual transition towards Markovianity and Gaussianity, in agreement with the results reported in Ref. [14]: as $D_{0}$ is increased, $\alpha \rightarrow 2$, the Gaussian value; $H_{\alpha} \rightarrow 1 / 2$, the diffusive value. And, consequently, $\beta \rightarrow 1$, the Markovian value.

To conclude, we point out some advantages of our method with respect to two methods commonly used in turbulent plasma simulations [7]. The first one models the tracer radial motion as a sequence of jumps separated by waiting-times. $\alpha$ is then estimated from the jump statistics, $\beta$, from the waiting times. The difficulty is hidden behind the definitions of "jump" and "waiting-time," which are hard to come about in turbulent systems since tracers are never at rest. In contrast, our method does not rely on any user-provided input. The second method initializes the 


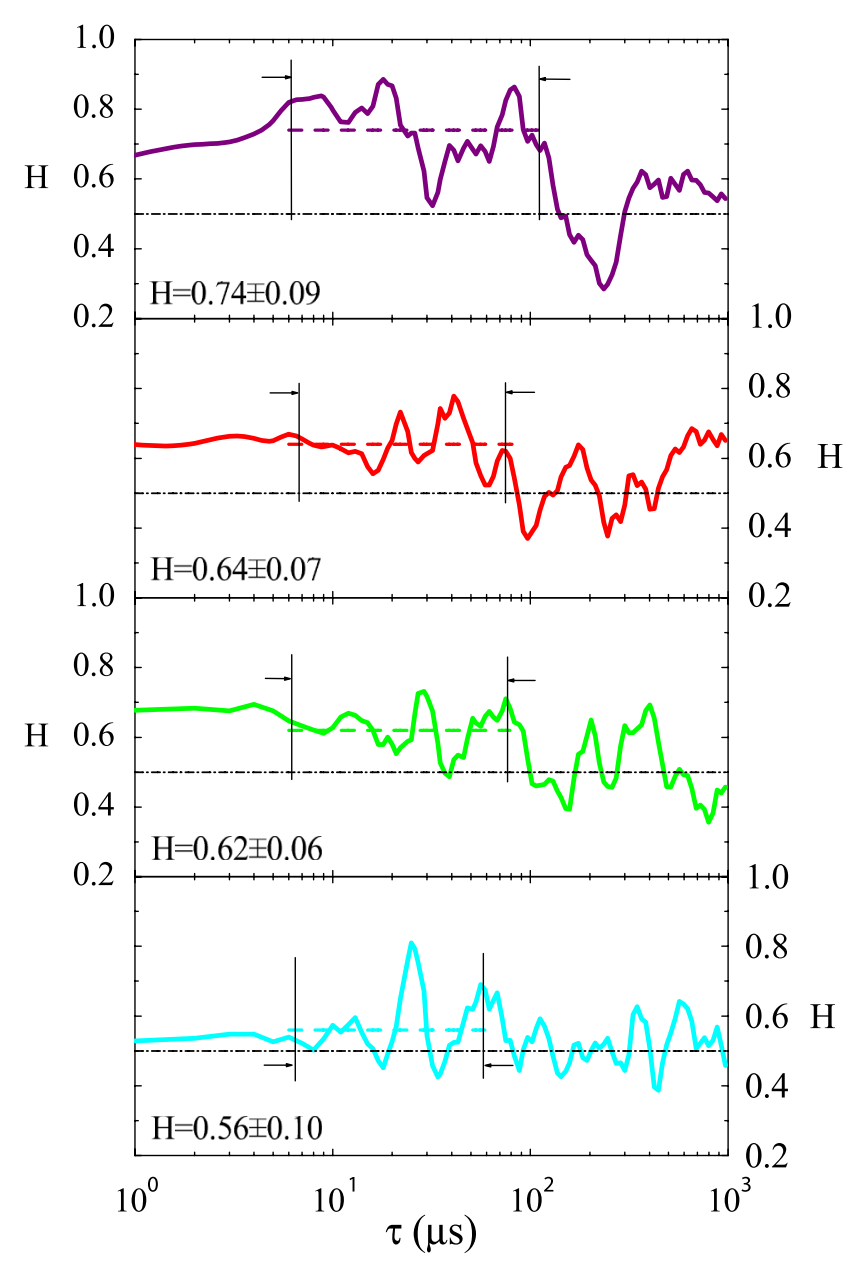

FIG. 3 (color online). $\quad \alpha$-Hurst exponent of Lagrangian velocity series versus elapsed time for various diffusivities (top: $D_{0}=$ 0; second: $D_{0}=0.07 \mathrm{~m}^{2} / \mathrm{s}$; third: $D_{0}=0.18 \mathrm{~m}^{2} / \mathrm{s}$; bottom: $D_{0}=1.19 \mathrm{~m}^{2} / \mathrm{s}$ ). The mesoscale is marked by arrows.

tracers in a narrow radial region and follows in time their radial distribution. Thus, it gives a numerical estimate of the propagator of the underlying transport process which, when compared with analytical propagators for various self-similar processes, yields $\alpha, \beta$, and $H_{\alpha}$. It is important to remark that the propagator and Lagrangian methods can be shown to be closely related in an infinitely large system. (In fact, in Ref. [9], $\alpha$ is introduced via an averaged propagator which turns into the prescription given here by exploiting self-similarity [11].) However, the fact that particles exit the boundaries makes the calculation of propagators in finite systems a delicate business, particularly in superdiffusive cases. A large number of tracers is needed. Additional techniques must be used to prevent finite-size distortions of the propagator tails which yield the exponents. In contrast, the Lagrangian method needs no additional processing and significantly less particles, since it reuses exiting ones to its advantage, which is not possible when computing propagators. This could be relevant for
TABLE I. Exponents $\alpha, H_{\alpha}, \beta$ and tracer confinement time.

\begin{tabular}{lcccr}
\hline \hline$D_{0}\left[\mathrm{~m}^{2} / \mathrm{s}\right]$ & $\alpha$ & $H_{\alpha}$ & $\beta$ & $\tau_{c}[\mu \mathrm{s}]$ \\
\hline 0 & $0.98 \pm 0.02$ & $0.74 \pm 0.09$ & $0.73 \pm 0.10$ & $130 \pm 5$ \\
0.07 & $1.39 \pm 0.02$ & $0.64 \pm 0.07$ & $0.89 \pm 0.11$ & $85 \pm 8$ \\
0.18 & $1.52 \pm 0.02$ & $0.62 \pm 0.06$ & $0.94 \pm 0.10$ & $72 \pm 7$ \\
1.19 & $1.73 \pm 0.02$ & $0.56 \pm 0.10$ & $0.97 \pm 0.18$ & $56 \pm 9$ \\
\hline \hline
\end{tabular}

applications to real flows, since the number of tracers that can be followed in experiments is limited.

Research supported by Spanish DGES Grant No. ENE2006-15244-C03-01/FTN and DOE Office of Science Grant No. DE-FG02-04ER54741 at University of Alaska. ORNL researchers sponsored by U.S. DOE under Contract No. DE-AC05-00OR22725.

*Corresponding author. jmier@fis.uc3m.es

[1] R. Metzler and J. Klafter, Phys. Rep. 339, 1 (2000); G. M. Zaslavsky, Phys. Rep. 371, 461 (2002); R. Metzler and J. Klafter, J. Phys. A 37, R161 (2004).

[2] I. Podlubny, Fractional Differential Equations (Academic Press, New York, 1998).

[3] K. Gentle et al., Phys. Plasmas 2, 2292 (1995); N. LopesCardozo, Plasma Phys. Controlled Fusion 37, 799 (1995); B. A. Carreras, IEEE Trans. Plasma Sci. 25, 1281 (1997).

[4] P. H. Diamond and T.S. Hahm, Phys. Plasmas 2, 3640 (1995); D. E. Newman, B. A. Carreras, P. H. Diamond, and T. S. Hahm, Phys. Plasmas 3, 1858 (1996).

[5] P. A. Politzer, Phys. Rev. Lett. 84, 1192 (2000); B. A. Carreras and B.P. van Milligen et al., Phys. Rev. Lett. 80, 4438 (1998); R. Sánchez, B. Ph. van Milligen, D. E. Newman, and B. A. Carreras, Phys. Rev. Lett. 90, 185005 (2003).

[6] B. A. Carreras, D.E. Newman, V.E. Lynch, and P. H. Diamond, Phys. Plasmas 3, 2903 (1996).

[7] B. A. Carreras, V. E. Lynch, and G. M. Zaslavsky, Phys. Plasmas 8, 5096 (2001); D. del-Castillo-Negrete, B. A. Carreras, and V. E. Lynch, Phys. Plasmas 11, 3854 (2004).

[8] T. Hauff and F. Jenko, Phys. Plasmas 13, 102309 (2006).

[9] R. Sánchez, B. A. Carreras, D. E. Newman, V. E. Lynch, and B. Ph. van Milligen, Phys. Rev. E 74, 016305 (2006).

[10] G. Falkovich, K. Gawedzki, and M. Vergassola, Rev. Mod. Phys. 73, 913 (2001).

[11] G. Samorodnitsky and M. S. Taqqu, Stable Non-Gaussian Processes (Chapman \& Hall, New York, 1994).

[12] H. E. Hurst, Trans. Am. Soc. Civ. Eng. 116, 770 (1951).

[13] B. Mandelbrot and J. van Ness, SIAM Rev. 10, 422 (1968).

[14] J. A. Mier, L. García, and R. Sánchez, Phys. Plasmas 13, 102308 (2006).

[15] B. A. Carreras, K. Sidikman, P. H. Diamond, P. W. Terry, and L. García, Phys. Fluids B 4, 3115 (1992).

[16] R. Sánchez, D. E. Newman, and B. A. Carreras, Nucl. Fusion 41, 247 (2001). 